\title{
Burnout als Krankheitskonzept
}

\author{
Burnout as a Disease Category
}

\author{
Bibliografie \\ DOI http://dx.doi.org/ \\ 10.1055/s-0031-1276895 \\ Psychiat Prax 2011; 38: \\ 320-322 \\ (c) Georg Thieme Verlag KC \\ Stuttgart · New York . \\ ISSN 0303-4259
}

Korrespondenzadressen

Dr. med. Axel Schüler-

Schneider

Guiollettstraße 27

60325 Frankfurt am Main

psymed@t-online.de

\section{Priv.-Doz. Dr.}

\section{Barbara Schneider}

Klinik für Psychiatrie, Psychosomatik und Psychotherapie Johann Wolfgang Goethe-

Universität

Heinrich-Hoffmann-Straße 10 60528 Frankfurt

B.Schneider@em.uni-frankfurt. de

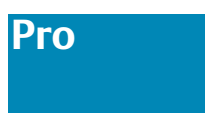

Burnout-Syndrom ist eine Sonderform der Depression mit ausgeprägter maniformer Abwehr

Freudenberger übertrug 1974 den Begriff Burnout aus der Kerntechnologie in den sozialwissenschaftlichen Bereich. Gerade die Menschen, die in einem schönen, abwechslungsreichen Beruf mit viel Verantwortung und mit sozialem Engagement arbeiten, sind burnoutgefährdet [1]. Burnout ist das Ergebnis der Imbalanz zwischen den Merkmalen und Möglichkeiten einer Person und den Merkmalen und Möglichkeiten seines Berufes. Die Häufigkeit beträgt etwa 20\% [2].

Burnout-Syndrom ist eine Sonderform der Depression mit einer maniformen Abwehr. Durch vermehrte Aktivität wird versucht das sichere Scheitern zu verhindern. Es ist ein Prozess, der unbehandelt in eine Depression mündet [3]. Es ist wichtig, die resultierende Erkrankung, nämlich die Depression nach ihrem Schweregrad mit einer Ziffer aus dem Kapitel F des ICD-10 zu verschlüsseln und nicht die Befindlichkeitsstörung nach Ziffer Z73.0. So ist auch eine längere Krankschreibung möglich. Dies alleine ist aber keine ausreichende Therapie. Nur eine Aufarbeitung der Konflikte führt zu einer Stabilisierung. Die aktuellen Konflikte haben in der Regel eine lange Vorgeschichte und stellen oft eine Reaktivierung früherer Konflikte dar.

Das erbliche Risiko für Depression beträgt $5 \%$. Wenn Depressionen in der Familie vorliegen erhöht sich das Risiko, an einer Depression zu erkranken auf $15 \%$, bei eineiigen Zwillingen liegt es sogar bei $50 \%$.

Eine Untersuchung der WHO kam zum Ergebnis, dass es zu einer 10-fachen Zunahme von Depressionen in Deutschland seit 1960 gekommen ist. Es gibt mehr als 4 Millionen behandlungsbedürftige Depressionskranke in Deutschland [4].

Der arbeitsplatzbezogene Stress nehme zu und damit auch die Häufigkeit von Burnout-Syndrom und Depression. Dabei werden die Betroffenen immer jünger. Seit 1945 ist die Suizidrate in Deutschland von über 20000 auf unter 10000 pro Jahr deutlich zurückgegangen [5]. Gleichzeitig haben die Suchterkrankungen zugenommen.

Burnout-Syndrom eignet sich als ein differenziertes Krankheitskonzept und sollte nur für Depressionen, die durch Konflikte am Arbeitsplatz ausgelöst wurden, verwendet werden.

Mittlerweile kam es zu einer Popularisierung des Begriffs Burnout-Syndrom und er wurde nicht nur für Erschöpfungszustände durch engagierte Arbeit, sondern u.a. auch bei Kindern, Schülern und für private Erschöpfungszustände verwendet. Dies führte zu einer Verwässerung des Begriffs. So entfernte er sich von einer klar umrissenen Diagnose, die notwendig ist, um eine geeignete Therapie festzulegen. Burnout-Syndrom sollte nur für berufsbedingte Erschöpfungs- und Depressionszustände Anwendung finden. Ein wichtiger Teil der Therapie besteht in der Entfernung aus dem Arbeitsprozess, dies ist im privaten Bereich schwierig.

\section{Pathophysiologie, Psychodynamik}

Unbehandelt verläuft der Burnout-Prozess in 4 Phasen und unterscheidet sich wesentlich von der üblichen kontinuierlich fortschreitenden Entwicklung der Depression. Durch vermehrte Aktivität, Anstrengung und emotionale Verausgabung versuchen die Betroffenen das unausweichliche Scheitern zu verhindern. Dies entspricht der manischen Abwehr einer drohenden Depression.

Phase 0 - Überlastungssituation

Phase 1 - manische Abwehr der Depression

Phase 2 - Flucht und Rückzug

Phase 3 - schwere Depression mit Isolation, Antriebslosigkeit, gedrückter Stimmung und oft auch suizidalen Gedanken und Suizidalität.

Früherkennung und Prophylaxe sind schwierig, da es sich um leistungsorientierte Menschen handelt, die es nicht gewohnt sind zu scheitern. Ge- 
fährdete Menschen sollten vorsorgen und sich regelmäßig selbst testen. Sport, Freizeitaktivitäten, Entspannungsverfahren und Stabilisierung der eigenen Persönlichkeit und psychischen Gesundheit tragen wesentlich dazu bei, dass der Übergang von einer Überlastung in eine Depression nicht erfolgen kann. Durch adäquate Behandlung ist Hilfe in allen Stadien möglich.

\section{Symptomatik}

Emotionale Erschöpfung liegt vor, wenn Wochenende und Urlaub nicht mehr zur Erholung ausreichen.

Depersonalisation ist der Verlust der Identität. Verhalten und Empfinden entsprechen nicht mehr der ursprünglichen Persönlichkeit. Ideale werden verraten. Dies ist verbunden mit einer gefühllosen, gleichgültigen, zynischen oder sarkastischen Einstellung gegenüber anderen Menschen. Diese werden abgelehnt, die Faust wird in der Tasche geballt, es knallt auch mal eine Tür. Selbst geliebte Partner oder Freunde werden unwirsch, aggressiv, ohne Verständnis behandelt. Soziale Kontakte werden gemieden. Es kommt zur Vernachlässigung von Hobbys und Privatleben. Oft ist durch den erhöhten zeitlichen und emotionalen Einsatz das soziale Netz lange nicht mehr gepflegt worden und daher nur noch brüchig vorhanden. So führt der depressive Rückzug nicht zur notwendigen Erholung. Der Abwärtstrend in die Depression wird verstärkt.

Abnehmende Leistungsfähigkeit tritt erst bei anhaltender Erschöpfung und Depersonalisation auf und führt zur schweren Depression mit Suizidalität.

Körperliche Symptome können alle Organsysteme im Sinne einer somatoformen Störung betreffen.

\section{Risikofaktoren und Prädisposition}

Frühkindliche, nicht verarbeitete Traumatisierungen wie frühe Verluste, Missbrauch, Gewalt in der Familie sind oft lange Zeit schlummernde Herde. Dies wird oft jahrzehntelang verdrängt, um nicht mit dem erlittenen Schmerz konfrontiert zu werden. Mit der abnehmenden Leistungsfähigkeit kommt es auch zu einer Schwächung der psychischen Abwehr und alte verdrängte Erinnerungen kommen wieder ins Bewusstsein. Das trägt zur Dekompensation bei.

Perfektionismus, Idealismus und übertriebene Identifikation mit dem Beruf, Zwanghaftigkeit, mangelnde Selbstreflexion, Versagensängste spielen eine wichtige Rolle bei der Entwicklung eines Burnout-Syndroms. Die Work-Life-Balance gerät aus dem Gleichgewicht, wenn der Arbeitsaufwand, insbesondere auch das emotionale Engagement über längere Zeit nicht ausreichend belohnt wird, sowohl materiell als auch emotional [6].

\section{Die spezielle Psychodynamik erfordert neues Behand-} lungskonzept: die Kurzzeit-Psychoanalyse

Als eine neue geeignete Therapieform entwickelte sich die Kurzzeit-Psychoanalyse. Die Behandlung erstreckt sich mit 3-4 Stunden pro Woche über 6-12 Wochen. Nach 4-6 Wochen sind die aktuellen und aktualisierten Konflikte geklärt, wenn auch nicht immer gelöst und die Familien- und Lebensgeschichte bei guter Compliance erarbeitet. $\mathrm{Zu}$ diesem Zeitpunkt kann eine realistische prognostische Einschätzung über den weiteren Verlauf erfolgen. Junge Menschen sind oft nach 6 Wochen schon wieder arbeitsfähig, bei älteren dauert es länger.

Wichtig ist die Entlastung von der Arbeit, um aus dem Abstand heraus über den eigenen Standpunkt, die Lebens- und Arbeitssituation, die Perspektiven, persönlichen Ziele, Visionen und bestehende Konflikte nachdenken zu können. Durch Bewegung und
Sport, mindestens 3-mal 30 min pro Woche wird der Körper gefordert, der Kreislauf stabilisiert und damit auch der Gedanke an die eigene Gesundheit gefördert. Durch Entspannung wie Qi Gong, die Urform aller Entspannungsverfahren, 2500 v. Chr. entwickelt, Autogenes Training, Progressive Muskelrelaxation, Tai Chi etc. wird die psychotherapeutische Arbeit unterstützt. Eine medikamentöse antidepressive Therapie ist zu Beginn der Behandlung sinnvoll.

\section{Kontra}

Immer mehr Menschen in unserer entgrenzten, hektischen und abgesehen von quartalsmäßig zu steigernden Umsatzzahlen ziellosen Welt identifizieren sich mit dem aktuell in den wissenschaftlichen $[1,7]$, mehr noch in den populären Medien heißdiskutierten Burnout-Phänomen, das einen chronischen berufsbedingten Überlastungszustand bzw. den dorthin führenden Prozess bezeichnen soll. Es treffe vor allem Hochengagierte und sei durch ein Versiegen individueller Ressourcen, eine Verringerung der kognitiven, emotionalen und sozialen Leistungsfähigkeit sowie der sukzessiven Manifestation psychischer und psychosomatischer Symptome gekennzeichnet. Berufliche und gesellschaftliche Faktoren werden als hauptverantwortlich angenommen.

Burnout spielt sich primär im Erleben Betroffener ab. Es ist kein Zufall, dass der Vater des Begriffs, Herbert Freudenberger [1], das Phänomen nicht als Ergebnis wissenschaftlicher Untersuchungen an seinen Patienten, sondern aus eigener Erfahrung nach langen Arbeitstagen als Psychotherapeut und bis weit nach Mitternacht als ehrenamtlich Tätiger heraus „entdeckt“ und publiziert hat. Dabei betonte er, dass Burnout keine psychische Erkrankung oder Neurose, sondern ein Phänomen eigener Art bzw. „nur“ das Ergebnis anhaltender Überforderung sei. „Burnout“, als vermeintlich selbsterklärendes Bild, wurde so zu einer Ersatzidentität von Betroffenen, die sich eben dieser Welt und ihren von außen und/oder innen determinierten Anforderungen nicht mehr gewachsen fühlen. Seelische Belastungen und Erkrankungen wurden dadurch stigmatisierungsarm kommunizierbar. Irgendwie „ausgebrannt“ ist schließlich jeder einmal! Dass Burnout als subjektives Krankheitsmodell derzeit offenkundig nötig und berechtigt ist, ist evident. Um den Begriff herum hat sich zwischenzeitlich ein blühendes Anti-Burnout-Business entwickelt. Von Kollegen, die als Burnout-Spezialisten ihren Patienten besonders nahe sein wollen, bis hin zum bunten Wellness-Markt, zwischen Vitamintabletten und Tango-Kuren: alle gehen anscheinend davon aus, dass Burnout Diagnosequalitäten hat. Nur formal-akribische Schreibtischpsychiater (wie wir), denen „Operationalisierung“ wichtiger ist als das Leid der Betroffenen, sehen das anders?

Operationalisierung ist die eine Seite der Medaille. Burnout ist als diagnostische Kategorie weder theoretisch, symptomatologisch noch psychometrisch unterbaut, entsprechend kennt ICD-10 den Begriff nur als - undefinierte - Zusatzcodierung. Die Demarkationslinien zu Störungen nach ICD-10 sind unscharf. Mehr als 130 potenzielle Burnout-Symptome werden diskutiert, von denen keines verpflichtend bzw. diagnosespezifisch sein soll [7]. Der Patient kann gewissermaßen alle davon haben oder auch fast keines. Auf eine von der Symptomatik ausgehende Operationalisierung von Burnout kann man somit verzichten, da es keinen verbindlichen diagnoseweisenden Logarithmus auf Symptomebene geben soll. Eine justiziable Verankerung von Burnout mit der Implikation damit eine Berufskrankheitskategorie zu bezeichnen, ist schon deshalb ausgeschlossen. Offen ist allerdings 
auch, ob „Burnout“ den Prozess oder den Endzustand eines solchen meint. Empirische Versuche, quasi gesetzmäßige BurnoutEntwicklungen, also vom Entflammt- zum Ausgebrannt-Sein, nachzuweisen, zeigen, dass es eben solche nicht gibt. Vielfach erleben sich bereits Berufsanfänger in Antizipation künftiger Belastungen als ausgebrannt. Absehbar bezeichnet Burnout damit ganz heterogene Phänomene. Dies erklärt auch, warum keine diese „Diagnose stützenden“ Biomarker identifiziert werden konnten [8].

Zentraler Anspruch etablierter psychiatrischer Diagnosesysteme ist es, diagnostische Kategorien objektiv und reliabel abzubilden. Es zeigte sich, dass hohe diagnostische Reliabilität nur durch Fokussierung auf deskriptiv erfassbare Symptome, bei gleichzeitigem Verzicht auf eben nicht reliabel abbildbare ätiologische Aspekte (z.B. Hypothesen zu neurotischer vs. endogener Genese von Depressionen) erreicht werden kann. ICD-10-Diagnosen des Kapitels „psychische Störungen“ sind somit aus der ExpertenPerspektive heraus definierte Kategorien, die per se nichts erklären und diesbezüglich bedürftige Betroffene schlicht im Regen stehen lassen. Burnout ist umgekehrt der Versuch, eine den Bedürfnissen leidender Menschen entgegenkommende Diagnose zu formulieren. Aus Burnout eine ICD-10 konforme Diagnose zu machen, abgesehen davon, dass es aus den skizzierten Gründen nicht funktionieren kann, wird das Problem zunehmender Distanz zwischen den eigenen Interessen folgenden Perspektiven von Experten und Betroffenen leider definitiv nicht lösen.

Gesetzt den Fall, unser Kontrahent hätte es tatsächlich geschafft: Burnout wäre eine operationalisierte ICD-11-Diagnose. Er säße nun einem Menschen gegenüber, der sich absolut „Burnout“ fühlt, die Kriterien aber nicht erfüllt: „Herr X., ich kann ihnen die erfreuliche Mitteilung machen, Sie sind gar nicht ausgebrannt! “ Wäre Herr X. dann wirklich erleichtert? Natürlich nicht, er würde sich abgrundtief unverstanden fühlen! Und das zurecht. Burnout hat seine fundamentale Berechtigung als subjekti- ves Krankheitsmodell. Wenn man daraus eine professionelle Diagnose macht, dann gibt man niemanden etwas, sondern nimmt Betroffenen ein wichtiges Kommunikationsmedium und möglicherweise Aspekte ihrer Identität!

Fassen wir zusammen: als aktuelles soziales Paradigma und Forschungsthema ist Burnout hochaktuell und unverzichtbar. Burnouterleben impliziert für Betroffene, die eigenen Stressbewältigungsstrategien zu reflektieren, Hilfe zu suchen, konstruktiv an der Passung zwischen Fähigkeiten, Einstellungen und Anforderungen zu arbeiten. Was wollen wir als Psychiater mehr? Aus Burnout eine operationalisierte Diagnose zu machen und damit das Phänomen im starren, potenziell stigmatisierenden ICDWeltbild zu verankern, das wäre nicht nur paradox, sondern genau das, was weder Herbert Freudenberger noch die Betroffenen wollen! Als Diagnose kann Burnout nicht funktionieren. Als subjektives Krankheitsmodell verweist der Begriff prägnant auf vitale Bedürfnisse und Nöte einer im Wandel begriffenen, existenziell verunsicherten Gesellschaft.

\section{Literatur}

1 Freudenberger $H$. Staff Burnout. J Social Issues 1974; 30: 159-165

2 Soler JK, Yaman H, Esteva $M$ et al. Burnout in European Family Doctors. EGPRN Study. Fam Pract 2008; 25: 245-265

3 Mentzos S. Lehrbuch der Psychodynamik. Göttingen: Vandenhoeck \& Ruprecht; 2009

4 Wilken UJ, Breucker G. Mental Health in the Workplace-Situation Analysis Germany. ILO Study. WHO; 2000

5 Reimer C, Trinkaus S, Jurkat HB. Suizidalität bei Ärztinnen und Ärzten. Psychiat Praxis 2005; 32: 381-385

6 Siegrist J. Adverse health effects of high effort - low reward conditions at work. Journal of Occupational Health Psychology 1996; 1: 27-43

7 Burisch M. Das Burnout-Syndrom. Theorie der Inneren Erschöpfung. Heidelberg: Springer; 2010

8 Danhof-Pont MB, van Veen T, Zitman FG. Biomarkers in Burnout: A Systematic Review. J Psychosom Res 2011; 70: 505-524 